\title{
II. Bases fisiopatológicas del entrenamiento muscular en pacientes con enfermedad pulmonar obstructiva crónica
}

\author{
FERNANDO SALDÍAS P.* y ORLANDO DÍAZ P.*
}

\author{
Pathophysiological bases of muscular training in patients with chronic obstructive \\ pulmonary disease
}

Dyspnea and decreased exercise capacity are the main factors that limit the daily living activities in patients with chronic respiratory diseases. The cardinal symptoms limiting exercise capacity in most patients with chronic obstructive pulmonary disease (COPD) are dyspnea and fatigue, which could be caused by alveolar ventilation and gas exchange disturbances, skeletal muscle dysfunction and/or cardiovascular failure. Anxiety, lack of motivation and depression were also associated with reduced exercise capacity, probably affecting the perception of symptoms. The relationship between psychological status and mood disorders in patients with COPD and exercise tolerance is complex and not yet fully understood. The origin of the exercise capacity limitation in COPD patients is multifactorial, so the separation of the variables involved for academic purposes is not always feasible. The pathogenic mechanisms may interact in complex ways, as an example, muscle deconditioning and hypoxemia can increase alveolar ventilation causing exercise limitation. Therefore, physical training and supplemental oxygen can reduce ventilatory limitation during exercise without changing lung function and maximum ventilatory capacity. The analysis of these factors could potentially identify reversible conditions that can improve the exercise performance and quality of life of patients with COPD, such as hypoxemia, bronchospasm, heart failure, arrhythmias, musculoskeletal dysfunction and myocardial ischemia. This review examines the principal mechanisms contributing to physical activity limitation in patients with COPD: alveolar ventilation and gas exchange abnormalities, cardiovascular and musculoskeletal system dysfunction, and respiratory muscles dysfunction.

Key words: Pulmonary rehabilitation; guidelines; chronic obstructive pulmonary disease; chronic lung disease; exercise; dyspnea; health-related quality of life; inspiratory muscles; supplemental oxygen; noninvasive ventilation; nutrition.

\section{Resumen}

La disnea y la disminución de la capacidad de realizar ejercicio son los principales factores que limitan las actividades de la vida diaria en pacientes con enfermedades respiratorias crónicas. Los síntomas cardinales que limitan la capacidad de ejercicio en la mayoría de los pacientes con enfermedad pulmonar obstructiva crónica (EPOC) son la disnea y/o fatigabilidad, los cuales pueden ser ocasionados por trastornos de la ventilación alveolar e intercambio gaseoso, disfunción de los músculos esqueléticos y/o falla cardiovascular. La ansiedad, falta de motivación y depresión también han sido asociadas a una menor capacidad de realizar ejercicio, probablemente afectando la percepción de los síntomas. La relación entre el estado psicológico y los trastornos del ánimo en pacientes con EPOC y la tolerancia al ejercicio es compleja y aún no ha sido completamente dilucidada. El origen de la limitación de la capacidad de ejercicio en pacientes con EPOC es multifactorial, por lo cual la separación de las variables involucradas con fines académicos no siempre es factible realizarlo en los pacientes. Los mecanismos patogénicos pueden relacionarse en forma compleja, a modo de ejemplo, el desacondicionamiento físico y la hipoxemia pueden contribuir a aumentar la ventilación alveolar

* Departamento de Enfermedades Respiratorias, Facultad de Medicina, Pontificia Universidad Católica de Chile. 
ocasionando limitación del ejercicio de causa ventilatoria. Por lo tanto, el entrenamiento físico y el suplemento de oxígeno pueden reducir la limitación ventilatoria durante el ejercicio sin modificar la función pulmonar o la capacidad ventilatoria máxima. El análisis de los factores limitantes de la capacidad de ejercicio permite identificar trastornos potencialmente reversibles que pueden mejorar la calidad de vida de los enfermos, tales como la hipoxemia, broncoespasmo, insuficiencia cardiaca, arritmias, disfunción musculoesquelética y/o isquemia miocárdica. En esta revisión se examinan los principales mecanismos que contribuyen a la limitación de la actividad física en pacientes con EPOC: anomalías de la ventilación alveolar e intercambio gaseoso, disfunción cardiovascular y del sistema músculo-esquelético y disfunción de los músculos respiratorios.

Palabras clave: Rehabilitación respiratoria; guías clínicas; enfermedad pulmonar obstructiva crónica; enfermedad respiratoria crónica; ejercicio; disnea; calidad de vida relacionada a salud; músculos inspiratorios; oxígeno suplementario; ventilación no invasiva; nutrición.

\section{Definición}

La Sociedad de Tórax Americana (ATS) y la Sociedad Europea de Respiratorio (ERS) han definido la rehabilitación respiratoria (RR) como una intervención multidisciplinaria basada en la evidencia en pacientes portadores de enfermedades respiratorias crónicas, que son sintomáticos y a menudo tienen disminuida su capacidad física, integrada en el tratamiento individualizado del paciente, con el propósito de reducir los síntomas, mejorar la capacidad funcional, aumentar la participación y reducir los costos asociados al cuidado de la salud, estabilizando o revirtiendo las manifestaciones sistémicas de la enfermedad ${ }^{1}$. Los programas de rehabilitación respiratoria incluyen la evaluación del paciente, ejercicios de entrenamiento muscular, educación y apoyo psicosocial ${ }^{1-3}$. En un sentido más amplio, la rehabilitación respiratoria abarca un espectro de estrategias de intervención integrados en el manejo a lo largo de la vida de los pacientes con enfermedades respiratorias crónicas, e involucra la participación y colaboración activa del paciente, su grupo familiar y el equipo de salud.

En esta definición destacan tres principios básicos de la rehabilitación respiratoria': a) Multidisciplinaria: El programa considera la participación de un equipo multiprofesional (médicos, enfermeras, terapistas respiratorio y ocupacional, psicólogos, especialistas en ejercicio, nutrición y otras especialidades de la salud) con distintos conocimientos, habilidades y destrezas integrados para resolver las necesidades particulares de cada paciente; b) Individual: Los pacientes con enfermedades respiratorias invalidantes requieren evaluación y tratamiento personalizado, los objetivos del programa deben ser realistas y centrados en las necesidades del individuo; c) Enfoque multisistémico: El programa debe abordar las necesidades físicas, emocionales, psicológicas y sociales de los pacientes, integrándose a las otras intervenciones de tratamiento y prevención, para mejorar la condición física y psicológica, función pulmonar y tolerancia al ejercicio de los enfermos.

\section{Disnea y limitación de la capacidad de ejercicio en la EPOC}

La disnea y la disminución de la capacidad de realizar ejercicio son los principales factores que limitan las actividades de la vida diaria en pacientes con enfermedades respiratorias crónicas ${ }^{4}$. Los síntomas cardinales que limitan la capacidad de ejercicio en la mayoría de los pacientes con enfermedad pulmonar obstructiva crónica (EPOC) son la disnea $\mathrm{y} / \mathrm{o}$ fatigabilidad, los cuales pueden ser ocasionados por trastornos de la ventilación alveolar e intercambio gaseoso, disfunción de los músculos esqueléticos y/o falla cardiovascular ${ }^{5}$. La ansiedad, falta de motivación y depresión también han sido asociadas a una menor capacidad de realizar ejercicio, probablemente afectando la percepción de los síntomas ${ }^{6,7}$. La relación entre el estado psicológico y los trastornos del ánimo en pacientes con EPOC y la tolerancia al ejercicio es compleja y aún no ha sido completamente dilucidada ${ }^{8}$.

El origen de la limitación de la capacidad de ejercicio en pacientes con EPOC es multifactorial, por lo cual la separación de las variables involucradas con fines académicos no siempre es factible realizarlo en los pacientes. Los mecanismos patogénicos pueden relacionarse en forma compleja, a modo de ejemplo, el desacondicionamiento físico y la hipoxemia pueden contribuir a aumentar la ventilación alveolar ocasionando limitación del ejercicio de causa ventilatoria. Por lo tanto, el entrenamiento físico y el suplemento de oxígeno pueden reducir la limitación ventila- 
toria durante el ejercicio sin modificar la función pulmonar o la capacidad ventilatoria máxima. El análisis de los factores limitantes de la capacidad de ejercicio permite identificar trastornos potencialmente reversibles que pueden mejorar la calidad de vida de los enfermos, tales como la hipoxemia, broncoespasmo, insuficiencia cardíaca, arritmias, disfunción musculoesquelética y/o isquemia miocárdica9 .

\section{Factores que contribuyen a la limitación de la capacidad de ejercicio en pacientes con EPOC}

\section{Anomalías de la ventilación alveolar}

En los pacientes con EPOC, la ventilación aumenta sobre lo esperado durante el ejercicio, debido a un aumento de la ventilación de espacio muerto, alteración del intercambio gaseoso (hipoxemia, hipercapnia) y aumento de la demanda ventilatoria asociada al desacondicionamiento físico y disfunción de los músculos periféri$\cos ^{5,10,11}$. Además, la ventilación máxima durante el ejercicio a menudo está limitada por factores mecánicos, de hecho, la limitación crónica del flujo aéreo disminuye el vaciamiento pulmonar durante la espiración tranquila ${ }^{12,13}$, fenómeno que se exacerba durante el ejercicio ${ }^{14}$. Esto ocasiona hiperinflación pulmonar dinámica ${ }^{15}$, aumento del trabajo respiratorio, aumento de la carga de los músculos respiratorios ${ }^{16,17}$ y percepción subjetiva de dificultad respiratoria (disnea).

\section{Anomalías del intercambio gaseoso}

La hipoxia tisular disminuye la resistencia a la fatiga y tolerancia al ejercicio en pacientes con EPOC por mecanismos directos e indirectos. La hipoxemia aumenta la ventilación alveolar estimulando directamente los quimiorreceptores periféricos ubicados en los cuerpos carotideos e indirectamente estimulando la producción de ácido láctico. La hiperlactacidemia contribuye a la disfunción muscular y aumenta la ventilación alveolar, ya que el efecto tampón del ácido láctico aumenta la producción de anhídrido carbónico ${ }^{18}$. El suplemento de oxígeno durante el ejercicio en pacientes con EPOC, con o sin hipoxemia crónica, permite aumentar la intensidad del entrenamiento físico, probablemente a través de varios mecanismos, entre los que se incluyen: a) el menor atrapamiento aéreo o disminución de la hiperinflación dinámica al descender la frecuencia respiratoria, b) disminución de la sobrecarga cardíaca y presión de la arteria pulmonar $y, c)$ menor producción de ácido láctico ${ }^{19-22}$.

\section{Disfunción cardiovascular}

El principal mecanismo que explica la disfunción cardiovascular en pacientes con EPOC es el aumento de la postcarga del ventrículo derecho ocasionado por la resistencia vascular pulmonar elevada debido a daño vascular directo ${ }^{23}$, vasoconstricción hipóxica ${ }^{24}$ y/o aumento de la resistencia vascular pulmonar efectiva por eritro$\operatorname{citosis}^{25}$. La sobrecarga del ventrículo derecho se asocia a hipertrofia ventricular y si no es tratada oportunamente, ocasiona falla ventricular dere$\mathrm{cha}^{26}$. La disfunción ventricular derecha puede comprometer el llene ventricular izquierdo por desviación del tabique interventricular, reduciendo la reserva cardíaca durante el ejercicio ${ }^{27}$.

Las taquiarritmias asociadas a la disfunción miocárdica, el atrapamiento aéreo y el consecuente aumento de la presión de la aurícula derecha pueden también afectar la función cardíaca durante el ejercicio ${ }^{28}$. En estas circunstancias, es posible que haya disfunción ventricular izquierda durante el ejercicio en pacientes con EPOC, lo que no se detecta en condiciones de reposo físico ${ }^{29}$.

Varios estudios han demostrado el efecto fisiológico favorable del ejercicio de alta intensidad $^{30-32}$, pero ha sido difícil determinar la contribución del sistema cardiovascular a la mejoría documentada de la función de los músculos periféricos. El efecto del entrenamiento muscular en el sistema cardiovascular de pacientes respiratorios crónicos ha sido sólo parcialmente definido y debería ser estudiado en mayor profundidad.

Finalmente, la inactividad ocasiona desacondicionamiento cardiovascular reduciendo la tolerancia al ejercicio. Es importante reconocer que una proporción significativa del aumento de la tolerancia al ejercicio después del entrenamiento físico probablemente se deba a mejoría de la función cardiovascular.

\section{Disfunción del sistema musculoesquelético}

En la Tabla 1 se describen las principales alteraciones del músculo esquelético en pacientes con afecciones respiratorias crónicas y los mecanismos fisiológicos que explican la mejoría después del entrenamiento muscular. La pérdida de peso y disminución de la masa muscular periférica se observa en alrededor de un 30\% de los pacientes ambulatorios con $\mathrm{EPOC}^{33}$. La disfunción de los músculos periféricos podría ser atribuible al desacondicionamiento físico inducido por la inactividad, inflamación sistémica, estrés oxidativo, alteraciones de los gases en sangre, uso de corticoides y reducción de la masa muscular ${ }^{34}$. El cuádriceps ha sido ampliamente estudiado en pacientes con EPOC por su accesibilidad y fun- 
Tabla 1. Alteraciones estructurales y bioquímicas del sistema musculoesquelético en la Enfermedad Pulmonar Obstructiva Crónica. Efectos del entrenamiento muscular

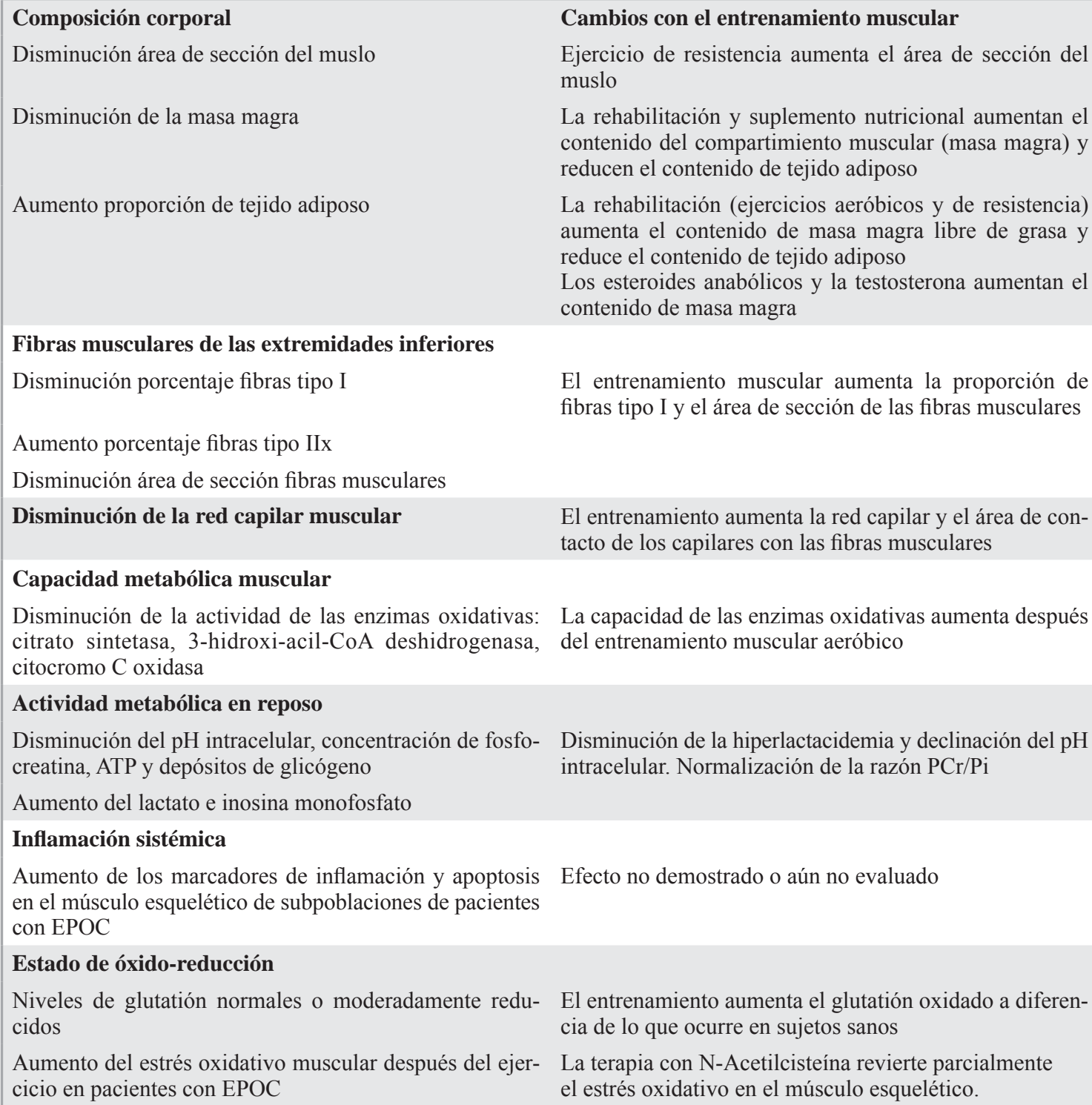

Disminución de la actividad de las enzimas oxidativas: La capacidad de las enzimas oxidativas aumenta después citrato sintetasa, 3-hidroxi-acil-CoA deshidrogenasa, del entrenamiento muscular aeróbico citocromo C oxidasa

\section{Actividad metabólica en reposo}

Disminución del pH intracelular, concentración de fosfocreatina, ATP y depósitos de glicógeno

Disminución de la hiperlactacidemia y declinación del pH intracelular. Normalización de la razón $\mathrm{PCr} / \mathrm{Pi}$

Aumento del lactato e inosina monofosfato

Inflamación sistémica

Aumento de los marcadores de inflamación y apoptosis en el músculo esquelético de subpoblaciones de pacientes con EPOC

\section{Estado de óxido-reducción}

Niveles de glutatión normales o moderadamente reducidos

El entrenamiento aumenta el glutatión oxidado a diferencia de lo que ocurre en sujetos sanos

Aumento del estrés oxidativo muscular después del ejercicio en pacientes con EPOC

Cambios con el entrenamiento muscular

Ejercicio de resistencia aumenta el área de sección del muslo

La rehabilitación y suplemento nutricional aumentan el contenido del compartimiento muscular (masa magra) y reducen el contenido de tejido adiposo

La rehabilitación (ejercicios aeróbicos y de resistencia) aumenta el contenido de masa magra libre de grasa y reduce el contenido de tejido adiposo

Los esteroides anabólicos y la testosterona aumentan el contenido de masa magra

El entrenamiento muscular aumenta la proporción de fibras tipo I y el área de sección de las fibras musculares

El entrenamiento aumenta la red capilar y el área de contacto de los capilares con las fibras musculares

La terapia con $\mathrm{N}$-Acetilcisteína revierte parcialmente el estrés oxidativo en el músculo esquelético.

ción en la deambulación, los hallazgos de los estudios no pueden ser generalizados a otros grupos musculares. Así, la fuerza y eficiencia mecánica de los músculos de las extremidades superiores al parecer estarían mejor preservadas, pero esto sigue siendo controversial ${ }^{35,36}$. De hecho, no existen estudios que hayan examinado simultáneamente biopsias musculares de las extremidades superiores e inferiores.

La capacidad aeróbica muscular disminuida podría afectar la tolerancia al ejercicio por varios mecanismos. El desarrollo de acidosis láctica frente a una carga de ejercicio determinada es un hallazgo frecuente en pacientes con $\mathrm{EPOC}^{37}$, 
La fatiga de las extremidades inferiores contribuye a la pobre tolerancia al ejercicio en pacientes respiratorios crónicos, siendo el principal síntoma limitante en algunos pacientes ${ }^{37,39}$. En un estudio realizado en pacientes que referían fatiga de piernas durante el ejercicio, la administración de ipratropio inhalado no aumentó la resistencia a la fatiga a pesar que mejoró un $11 \%$ el $\mathrm{VEF}_{1}$, destacando la importancia de la disfunción muscular periférica en la intolerancia al ejercicio en algunos pacientes con $\mathrm{EPOC}^{40}$.

\section{Disfunción de los músculos respiratorios}

El diafragma de los pacientes con EPOC se adapta a la sobrecarga crónica y aumenta la resistencia a la fatiga ${ }^{41,42}$. Así, a idéntico volumen pulmonar, los músculos inspiratorios son capaces de generar más fuerza comparado con sujetos controles sanos ${ }^{43,44}$. Esto ocurre precozmente en el curso de la enfermedad, incluso antes que se produzcan adaptaciones en el músculo esqueléti$\mathrm{Co}^{45}$. Sin embargo, estos pacientes a menudo tienen hiperinflación, lo cual coloca a sus músculos inspiratorios en desventaja mecánica. A pesar de los cambios adaptativos que ocurren en el diafragma, la fuerza y tolerancia a la fatiga de los músculos respiratorios está reducida en pacientes con EPOC ${ }^{46,47}$. Por ello, la debilidad muscular respiratoria objetivada midiendo las presiones respiratorias máximas suele estar presente en estos $\operatorname{casos}^{46-48}$. La disfunción de los músculos respiratorios contribuye a la retención de $\mathrm{CO}_{2}{ }^{49}$, disnea $^{50}$, hipoxemia nocturna ${ }^{51}$ y reducción de la tolerancia al ejercicio ${ }^{37}$.

Los pacientes con EPOC utilizan durante el ejercicio una mayor proporción de su presión inspiratoria máxima comparado con sujetos sanos, probablemente debido al aumento de la carga de los músculos respiratorios por la hiperinflación dinámica ${ }^{52}$. Otro factor limitante del ejercicio es el aumento de la resistencia vascular sistémica al aumentar la carga sobre el diafrag$\mathrm{ma}^{53}$, lo cual podría determinar la substracción de flujo sanguíneo de los músculos periféricos para suplir las necesidades del diafragma. Sin embargo, esta hipótesis aún no ha sido demostrada.

\section{Estrategias para reducir la hiperinflación dinámica y aumentar la tolerancia al ejercicio en pacientes con EPOC}

La disnea y limitación de la capacidad física en pacientes portadores de EPOC es multifactorial, relacionada a variables respiratorias (hipoxe- mia, hiperinflación dinámica, fatiga muscular) y sistémicas (malnutrición, disfunción muscular y cardiovascular, inflamación, comorbilidades $)^{5,10-13}$. La relación que existe entre la gravedad de la obstrucción bronquial y la limitación de la actividad física ha determinado la búsqueda de estrategias que permitan reducir la hiperinflación pulmonar y por ende mejorar la tolerancia al ejercicio en pacientes con EPOC. Se han descrito cuatro intervenciones farmacológicas y no farmacológicas que reducen la hiperinflación dinámica durante el ejercicio y aumentan la tolerancia al ejercicio:

\section{Terapia broncodilatadora}

Los agentes broncodilatadores (agonistas $\beta 2$ adrenérgicos y anticolinérgicos inhalados) son la principal opción farmacológica para reducir la hiperinflación dinámica durante el ejercicio ${ }^{54-56}$. En un estudio multicéntrico se examinó el efecto de tiotropio $18 \mu \mathrm{g} /$ día versus placebo en 198 pacientes con EPOC moderada a grave tratados durante seis semanas, la tolerancia al ejercicio mejoró significativamente y disminuyó la hiperinflación dinámica en el grupo tratado con tiotropio $^{55}$. Al final de las seis semanas, los pacientes tratados con tiotropio eran capaces de ejercitarse por un período de tiempo más prolongado que el grupo placebo (incremento de la duración del ejercicio promedio: $21 \%$ ). Así, los broncodilatadores inhalados mejoran la tolerancia al ejercicio al disminuir la resistencia de la vía aérea y la hiperinflación dinámica, reduciendo la magnitud de la disnea para un determinado nivel de ejercicio.

\section{Suplemento de oxígeno}

El tratamiento con oxígeno suplementario en pacientes hipoxémicos con EPOC ha demostrado que mejora la tolerancia al ejercicio, disminuye las complicaciones y prolonga la sobrevida ${ }^{57}$. Existe evidencias que apoyan su empleo en pacientes sin hipoxemia ${ }^{21,58,59}$, el oxígeno suplementario durante el ejercicio de alta intensidad reduce el estímulo ventilatorio, y por ende la frecuencia respiratoria, prolongando el tiempo espiratorio y reduciendo la hiperinflación dinámica, mejorando la tolerancia al ejercicio. En un estudio realizado en pacientes con EPOC sin hipoxemia de ejercicio a carga constante en cicloergómetro con diferentes fracciones inspiradas de oxígeno, los autores comunicaron reducción significativa de la hiperinflación dinámica cuando respiraron mezclas gaseosas con 30 y $50 \%$ de oxígeno durante la prueba versus aquellos que respiraron aire ambiente ${ }^{21}$. La hiperoxia disminuyó la ventilación alveolar a expensas de la frecuencia respiratoria, 
reduciendo la hiperinflación dinámica y prolongando el tiempo de ejercicio.

\section{Inhalación de mezcla gaseosa de oxígeno-helio}

La mecánica pulmonar en pacientes con EPOC con limitación ventilatoria puede mejorar respirando una mezcla de gases de baja densidad como el heliox ( $79 \%$ helio y $21 \%$ oxígeno). La inhalación de heliox reduce el flujo turbulento en pacientes con obstrucción bronquial cuando se incrementa la ventilación durante el ejercicio, lo cual aumenta la tolerancia al ejercicio y la ventilación minuto máxima ${ }^{60,61}$. Los pacientes respirando heliox duplicaron el tiempo de ejercicio a una carga constante en cicloergómetro $\mathrm{y}$ aumentaron significativamente la ventilación minuto al final del ejercicio comparado con el grupo control respirando aire ambiente ${ }^{60}$. A igualdad de tiempo, la capacidad inspiratoria aumentó alrededor de $200 \mathrm{~mL}$ con heliox, y se mantuvo elevada en el ejercicio máximo comparado con el grupo control. La inhalación de heliox actúa del mismo modo que los fármacos broncodilatadores, disminuyendo la resistencia al flujo y la hiperinflación dinámica durante el ejercicio, con lo cual aumenta la tolerancia al ejercicio.

\section{Rehabilitación respiratoria}

De todas las intervenciones disponibles en pacientes con EPOC, los programas de rehabilitación respiratoria que incluyen ejercicios de las extremidades inferiores son los que logran mayor mejoría de la tolerancia al ejercicio ${ }^{62}$. El entrenamiento muscular progresivo disminuye el estímulo ventilatorio para una actividad determinada reduciendo la producción de lactato muscular, aumenta la resistencia a la fatiga muscular y disminuye la hiperinflación dinámica; lo cual aumenta la tolerancia al ejercicio en pacientes con limitación crónica del flujo espiratorio ${ }^{63}$. En un estudio de entrenamiento muscular de alta intensidad en cicloergómetro tres veces por semana durante 7 semanas, en el examen cardiopulmonar a carga constante realizada a $75 \%$ del trabajo máximo, en el grupo entrenado disminuyó la ventilación minuto alrededor de $2 \mathrm{~L} / \mathrm{min}$ y la frecuencia respiratoria 3 ciclos/min y la capacidad inspiratoria aumentó en promedio $130 \mathrm{~mL}$ comparado con el grupo control, aumentando significativamente la resistencia a la fatiga y la tolerancia al ejercicio ${ }^{63}$.

El entrenamiento muscular disminuye el estimulo ventilatorio y la frecuencia respiratoria durante el ejercicio, prolongando el tiempo espiratorio, con lo cual disminuye la hiperinflación dinámica y prolonga el tiempo de ejercicio. El entrenamiento muscular asociado a broncodilatadores inhalados o suplemento de oxígeno permite aumentar la intensidad del ejercicio y prolongar su duración, obteniendo mayor beneficio de los programas de rehabilitación respiratoria ${ }^{20,64}$.

\section{Limitación del ejercicio y disfunción muscular en la EPOC: bases de la rehabilitación pulmonar}

La enfermedad pulmonar obstructiva crónica es una afección primariamente pulmonar con manifestaciones sistémicas asociadas como epifenómeno tales como la cardiopatía isquémica y trastornos del ánimo, o secundarias como la caquexia y pérdida de masa magra (parcialmente debido al aumento del trabajo respiratorio), osteoporosis por inmovilidad, disfunción autonómica por hipoxemia y disfunción de los músculos esqueléticos ${ }^{5,10,11,34}$. La disfunción muscular contribuye a las manifestaciones clínicas (disnea, fatigabilidad) que limitan el ejercicio y es un factor determinante de la menor tolerancia al ejercicio de los pacientes con EPOC ${ }^{10,34,35}$. Además, la debilidad muscular estaría relacionada, independiente de la función pulmonar, con un peor estado de salud ${ }^{65}$, mayor utilización de los recursos sanitarios ${ }^{66}$ y riesgo de muerte ${ }^{67}$. De este modo, la rehabilitación respiratoria y el entrenamiento muscular permitirían revertir la disfunción muscular, aumentar la tolerancia al ejercicio y mejorar la calidad de vida de los enfermos sin modificar la función pulmonar.

Una proporción significativa de los pacientes con EPOC avanzado desarrollan un estado de caquexia con importante pérdida de peso y masa muscular. Schols y cols, han reportado que hasta un $45 \%$ de los pacientes con EPOC estable con indicación de rehabilitación respiratoria tienen bajo peso y reducción de la masa magra ${ }^{33}$. El área de sección del muslo y la pantorrilla están disminuidos en pacientes con EPOC comparado con controles normales ${ }^{35,68}$. Las alteraciones estructurales y funcionales de los músculos esqueléticos de las extremidades inferiores en pacientes con EPOC descritas en la Tabla 1 contribuyen a los síntomas reportados por los enfermos y a su menor capacidad de realizar ejercicio ${ }^{34,35}$. La disnea es el principal factor limitante en la prueba de ejercicio incremental en treadmill, pero un $25 \%$ de los pacientes se detienen por fatiga de las extremidades inferiores, lo cual aumenta en la prueba de ejercicio en cicloergómetro ${ }^{69}$.

Los estudios fisiológicos realizados en pacientes con EPOC han demostrado disminución de 
la fuerza y tolerancia a la fatiga de los músculos cuádriceps comparado con sujetos normales s $^{35,70-72}$. Mientras que la fuerza y resistencia a la fatiga del diafragma, músculos abdominales y de las extremidades superiores se mantienen relativamente indemnes comparado con controles sanos ${ }^{48,70,73}$. En comparación con el cuádriceps, el diafragma es relativamente resistente a la fatiga con el ejercicio de alta intensidad y la ventilación voluntaria máxima en pacientes con $\mathrm{EPOC}^{74,75}$, y las fibras musculares aisladas del diafragma serían metabólicamente más eficientes comparado con controles normales ${ }^{76}$.

La disfunción muscular de extremidades inferiores es clínicamente significativa en pacientes con EPOC. La reducción de la masa muscular medida por métodos radiológicos y la fuerza de los cuádriceps son predictores de mortalidad ${ }^{67,77}$. La debilidad de los cuádriceps se ha asociado a menor distancia recorrida y actividad diaria ${ }^{78}$, menor consumo de oxígeno máximo ${ }^{50}$ y mayor utilización de los servicios de salud ${ }^{66}$. Las principales anomalías estructurales observadas en las biopsias musculares de los cuádriceps de pacientes con EPOC son la disminución de las fibras tipo I y aumento proporcional de las fibras tipo IIa y IIx, atrofia generalizada de las fibras musculares y reducción de la red capilar muscular ${ }^{79-82}$. En la Figura 1 se describe el círculo vicioso que limita la capacidad de ejercicio en los pacientes con disfunción de los músculos esqueléticos de las extremidades inferiores.

La actividad de las enzimas musculares oxidativas está disminuida en pacientes con EPOC, mientras que los estudios comunican resultados variables para la actividad de las enzimas glicolíticas $^{37,83}$. La capacidad de ejercicio de los músculos del muslo se correlaciona directamente con la proporción de fibras tipo I y la relación entre la actividad de las enzimas oxidativas y glicolíticas del músculo ${ }^{84}$. La disminución de la capacidad oxidativa y el cambio a metabolismo anaeróbico o glicolítico tienen efectos deletéreos sobre la capacidad de ejercicio, así el aumento del lactato sanguíneo es responsable de la caída del pH muscular y acidosis sistémica ${ }^{37,85}$. El nivel de lactato en sangre venosa femoral no se correlaciona con el transporte de oxígeno ni el flujo sanguíneo de las extremidades inferiores, lo cual apoya la presencia de anomalías bioquímicas en los músculos de los pacientes con $\mathrm{EPOC}^{37}$. De hecho, la depleción de fosfocreatina durante el ejercicio es mayor y la recuperación después del ejercicio es más lenta en pacientes con EPOC comparado con sujetos sanos ${ }^{86}$.

\section{Etiología de la disfunción muscular en la EPOC}

La disfunción muscular en pacientes con EPOC es multifactorial (Figura 2), la importancia relativa de los factores locales y sistémicos está siendo ampliamente investigada, los principales factores involucrados son:

\section{Hipoxia}

La hipoxia intermitente y crónica en pacientes con EPOC tiene efectos deletéreos sobre la función muscular: la proporción de fibras tipo I, el diámetro de sección de las fibras musculares, la

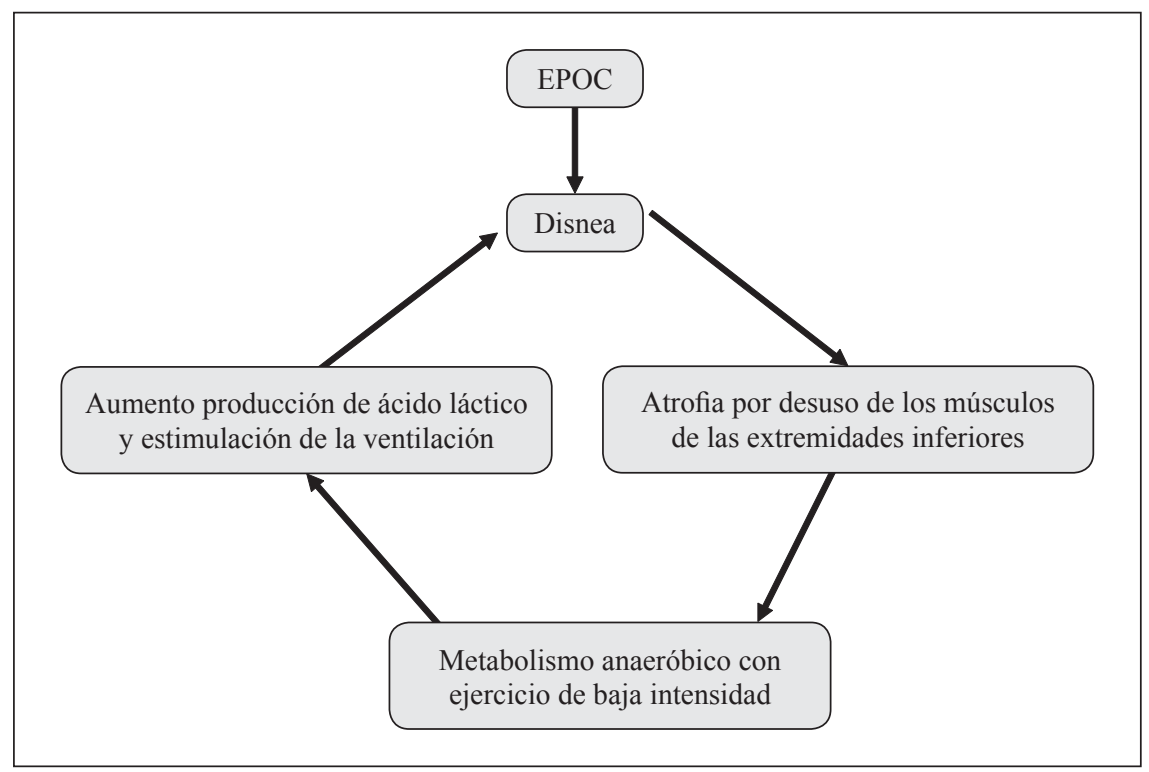

Figura 1. Círculo vicioso que limita la capacidad de ejercicio en los pacientes con EPOC y disfunción de los músculos esqueléticos de las extremidades inferiores. 


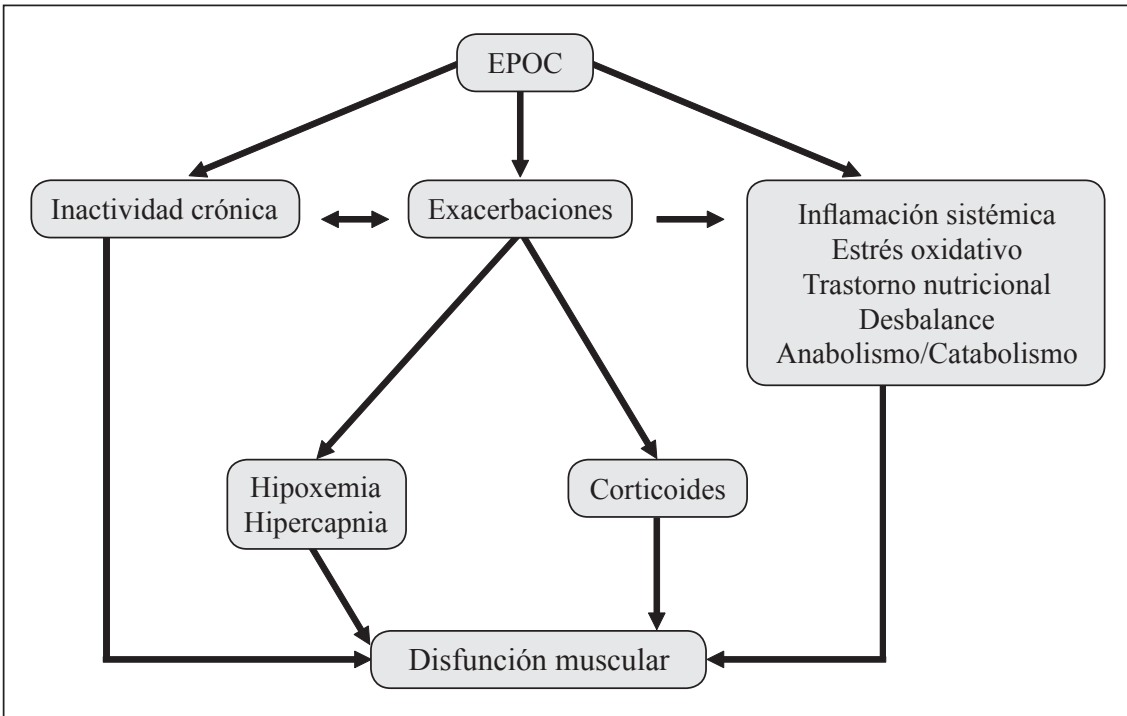

Figura 2. Etiología de la disfunción muscular en la enfermedad pulmonar obstructiva crónica. fuerza y resistencia a la fatiga disminuyen concomitantemente con la reducción de la actividad de las enzimas del ciclo de Krebs, oxidación de ácidos grasos y cadena respiratoria ${ }^{87-90}$. La concentración de ATP, glicógeno y fosfocreatina disminuye significativamente en pacientes con EPOC e insuficiencia respiratoria crónica comparado con pacientes sin falla respiratoria ${ }^{79}$. La hipoxia puede contribuir a la emaciación muscular por varios mecanismos: disminución de la producción de hormonas anabólicas, aumento de citoquinas proinflamatorias y generación de especies reactivas a oxígeno que contribuyen al estrés oxidativo ${ }^{91-93}$. La suplementación de oxígeno mejora parcialmente la contractilidad muscular de los pacientes con EPOC e insuficiencia respiratoria y aumenta el metabolismo aeróbico ${ }^{94}$.

\section{Hipercapnia}

La hipercapnia aguda se asocia a acidosis intracelular afectando el metabolismo muscular, disminuyendo la concentración de ATP, fosfocreatina y nucleótidos de adenosina ${ }^{95,96}$. La hipercapnia aguda disminuye la contractilidad del diafragma y músculos de las extremidades inferiores en sujetos sanos ${ }^{97,98}$. La coexistencia de hipercapnia con hipoxemia e hiperinflación dinámica en pacientes con EPOC dificulta el estudio de la relación que existe entre las alteraciones del intercambio gaseoso y la disfunción musculoesquelética.

\section{Corticosteroides}

Los pacientes con EPOC suelen ser tratados con corticoides sistémicos durante las exacerbaciones y ocasionalmente son mantenidos con dosis bajas durante períodos prolongados. Los corticoides sistémicos en dosis elevadas disminuyen la fuerza y resistencia a la fatiga de los músculos respiratorios y de las extremidades, lo cual revierte al reducir las dosis de esteroides ${ }^{99,100}$. La dosis y duración del tratamiento esteroidal que ocasiona disfunción muscular clínicamente significativa en pacientes con EPOC aún es motivo de controversia ${ }^{101}$. De hecho, es difícil separar el efecto de los esteroides de la inflamación y reducción de la actividad asociada a la exacerbación.

\section{Depleción nutricional}

La desnutrición se ha asociado a disminución de la fuerza y resistencia a la fatiga de las extremidades superiores e inferiores ${ }^{102,103}$. La pérdida de masa magra se ha asociado a menor capacidad de generar fuerza de los músculos esqueléticos $\mathrm{y}$ es mejor predictor de sobrevida en pacientes con EPOC que el peso corporal ${ }^{77}$. Los suplementos nutricionales no han demostrado efectos beneficiosos desde el punto de vista clínico y su efectividad en los programas de rehabilitación respiratoria no ha sido claramente demostrada ${ }^{104}$.

\section{Desbalance de hormonas anabólicas/catabólicas}

La estrecha relación que existe entre la disfunción muscular y pérdida de masa magra ha promovido el estudio del balance entre las hormonas anabólicas y catabólicas en pacientes con EPOC. Se ha observado una reducción de las hormonas anabólicas (testosterona, factor de crecimiento tipo insulina-1) en pacientes con EPOC, sin embargo, no se ha demostrado fehacientemente un desbalance en favor del catabolismo ${ }^{105,106}$. La 
administración de fármacos anabólicos para promover la síntesis proteica (testosterona, hormona del crecimiento, IGF-1) aumenta discretamente la masa y fuerza muscular ${ }^{107,108}$. El efecto de la terapia anabólica en la capacidad de ejercicio es menos significativo y no se recomienda su uso rutinario en los programas de rehabilitación respiratoria ${ }^{1-3}$.

\section{Inflamación sistémica}

La EPOC se asocia a un estado de inflamación sistémica de bajo grado, los niveles séricos de factor de necrosis tumoral alfa (TNF $\alpha)$ están elevados en pacientes que no logran ganar peso durante los programas de rehabilitación y suplementación nutricional ${ }^{109}$. Los niveles sanguíneos de interleuquina (IL) 6 y 8 , TNF $\alpha$ y proteína C reactiva en pacientes con EPOC se han asociado a mayor gasto energético en reposo, dando sustento a la hipótesis que las citoquinas proinflamatorias jugarían un papel en la caquexia asociada a la $\mathrm{EPOC}^{110,111}$. Los niveles elevados de IL-6 se han asociado a pérdida de masa magra y emaciación muscular en $\mathrm{EPOC}^{105}$. La inflamación sistémica asociada a las exacerbaciones agudas también podría ser relevante, Spruit y cols, observaron una relación inversa significativa entre los niveles de IL-8 y fuerza del cuádriceps en pacientes admitidos por una exacerbación de la $\mathrm{EPOC}^{112}$. El efecto de la inflamación sistémica en la función muscular aún es motivo de controversia y no permitiría explicar la indemnidad funcional de algunos grupos musculares como el diafragma y los músculos de la cintura escapular.

\section{Estrés oxidativo}

Los radicales libres tales como el anión superóxido y óxido nítrico pueden ocasionar daño oxidativo a los lípidos y proteínas de las membranas celulares, alterando la actividad de la cadena respiratoria de las mitocondrias. El aumento del estrés oxidativo ha sido demostrado durante las exacerbaciones agudas y el ejercicio, pero también ha sido documentado en la fase estable de la enfermedad ${ }^{113-115}$. El tratamiento antioxidante con $\mathrm{N}$-Acetilcisteína ha demostrado que reduce el estrés oxidativo inducido por el ejercicio y aumenta la resistencia a la fatiga del cuádriceps ${ }^{116}$. No está claro si el estrés oxidativo produce directamente disfunción muscular en pacientes con EPOC o forma parte de un epifenómeno del proceso de daño-reparación.

\section{Susceptibilidad genética}

La fuerza de los cuádriceps disminuye alrededor de un $30 \%$ en pacientes con EPOC moderada-grave comparado con controles sanos pareados por edad y género. Sin embargo, se ha observado considerable superposición entre ambos grupos, lo cual sugeriría que algunos pacientes serían particularmente susceptibles a desarrollar disfunción muscular. Estudios en gemelos sanos han demostrado que existen factores genéticos relacionados con la masa y fuerza muscular ${ }^{117}$. Estudios de polimorfismo de la enzima convertidora de angiotensina ${ }^{118}$ y el receptor de vitamina $\mathrm{D}^{119}$ se han relacionado con el desarrollo del sistema musculoesquelético y fuerza muscular. El estudio de los factores genéticos es incipiente y no permite aún obtener conclusiones.

\section{Inactividad y reducción de la actividad física diaria}

El compromiso preferencial de algunos grupos musculares sugiere que factores locales estarían involucrados en la disfunción muscular de la EPOC. Quizás el factor local más obvio sea la inactivi$\mathrm{dad}$, es razonable asumir que los pacientes con afecciones respiratorias crónicas sean más sedentarios; de hecho, los pacientes con EPOC realizan menos actividad física en la vida diaria y menor actividad de las extremidades inferiores ${ }^{120-122}$. Por otro lado, la actividad física regular de intensidad moderada disminuye la declinación acelerada de la función pulmonar y el riesgo de desarrollar EPOC en fumadores ${ }^{123}$. Además, en pacientes con EPOC el ejercicio físico disminuye el riesgo de hospitalización y la mortalidad global y de causas respiratorias ${ }^{124}$. Las evidencias que sustentan la contribución de la inactividad a la disfunción muscular de los pacientes con EPOC son: a) La debilidad muscular afecta predominantemente a las extremidades inferiores, con relativa indemnidad de las extremidades superiores y el diafragma ${ }^{35,48}$; b) Las características morfológicas y metabólicas del cuádriceps difieren de otros grupos musculares en la EPOC ${ }^{41,42}$. En el deltoides y el diafragma está aumentada la capacidad oxidativa, y también aumenta la proporción de fibras lentas tipo I en el diafragma de pacientes con EPOC grave; c) Las alteraciones morfológicas y funcionales del cuádriceps de pacientes con EPOC son similares a las observadas en sujetos sanos con desacondicionamiento físico o reposo prolongado ${ }^{125}$.

\section{Bibliografía}

1.- $\quad$ NICI L, DONNER C, WOUTERS E, ZUWALLACK R, AMBROSINO N, BOURBEAU J, et al. American Thoracic Society/European Respiratory Society Statement on Pulmonary Rehabilitation. Am J Respir Crit Care Med 2006; 173: 1390-1413.

2.- BRITISH THORACIC SOCIETY Standards of Care 
Subcommittee on Pulmonary Rehabilitation. Pulmonary Rehabilitation. Thorax 2001; 56: 827-34.

3.- RIES A L, BAULDOFF G S, CARLIN B W, CASABURI R, EMERY C F, MAHLER D A, et al. Pulmonary Rehabilitation. Chest 2007; 131: 4S-42S.

4.- MAHLER D A, BAIRD J C. Dyspnea in the elderly. In: Mahler DA, O’Donnell DE, editors. Dyspnea: mechanisms, measurement, and management, $2^{\text {nd }}$ ed. New York: Taylor \& Francis; 2005. pp. 19-28.

5.- O'DONNELL D E, BANZETT R B, CARRIERIKOHLMAN V, CASABURI R, DAVENPORT P W, GANDEVIA S C, et al. Pathophysiology of dyspnea in chronic obstructive pulmonary disease. A roundtable. Proc Am Thorac Soc 2007; 4: 145-68.

6.- JANSON C, BJORNSSON E, HETTA J, BOMAN G. Anxiety and depression in relation to respiratory symptoms and asthma. Am J Respir Crit Care Med 1994; 149: 930-4.

7.- $\quad$ PAREKH P I, BLUMENTHAL J A, BABYAK M A, MERRILL K, CARNEY R M, DAVIS R D, et al. Psychiatric disorder and quality of life in patients awaiting lung transplantation. Chest 2003; 124: 1682-8.

8.- BORAK J, CHODOSOWSKA E, MATUSZEWSKI A, ZIELINSKI J. Emotional status does not alter exercise tolerance in patients with chronic obstructive pulmonary disease. Eur Respir J 1998; 12: 370-3.

9.- WEISMAN I M, ZEBALLOS R J. An integrative approach to cardiopulmonary exercise testing. In: Weisman IM, Zeballos RJ, editors. Clinical exercise testing. Prog Respir Res Basil, Switzerland: Karger 2002; 32: 300-22.

10.- GRAZZINI M, STENDARDI L, GIGLIOTTI F, SCANO G. Pathophysiology of exercise dyspnea in healthy subjects and in patients with chronic obstructive pulmonary disease (COPD). Respir Med 2005; 99 : 1403-12.

11.- O'DONNELL D E. Breathlessness in patients with chronic airflow limitation. Chest 1994; 106: 904-12.

12.- HYATT R E. Expiratory flow limitation. J Appl Physiol 1983; 55: 1-7.

13.- PRIDE N B, MACKLEM P T. Lung mechanics in disease. In: Fishman AP, editor. Handbook of physiology. Bethesda, MD: Oxford University Press. American Physiological Society. pp. 659-92.

14.- JOHNSON B D, WEISMAN I M, ZEBALLOS R J, BECK K C. Emerging concepts in the evaluation of ventilatory limitation to exercise: the exercise tidal volume loop. Chest 1999; 116: 488-503.

15.- O'DONNELL D E, REVILL S M, WEBB K A. Dynamic hyperinflation and exercise intolerance in chronic obstructive pulmonary disease. Am J Respir Crit Care Med 2001; 164: 770-7.

16.- ALIVERTI A, STEVENSON N, DELLACA R L, LO M A, PEDOTTI A, CALVERLEY P M. Regional chest wall volumes during exercise in chronic obstructive pulmonary disease. Thorax 2004; 59: 210-6.
17.- DÍAZ O, VILLAFRANCA C, GHEZZO H, BORZONE G, LEIVA A, MILIC-EMILI J, LISBOA C. Role of inspiratory capacity on exercise tolerance in COPD patients with and without tidal expiratory flow limitation at rest. Eur Respir J 2000; 16: 269-75.

18.- SOMFAY A, PORSZASZ J, LEE S M, CASABURI R. Effect of hyperoxia on gas exchange and lactate kinetics following exercise onset in nonhypoxemic COPD patients. Chest 2002; 121: 393-400.

19.- O'DONNELL D E, D`ARSIGNY C, WEBB K A. Effects of hyperoxia on ventilatory limitation in advanced COPD. Am J Respir Crit Care Med 2001; 163 : 892-8.

20.- EMTNER M, PORSZASZ J, BURNS M, SOMFAY A, CASABURI R. Benefits of supplemental oxygen in exercise training in nonhypoxemic COPD patients. Am J Respir Crit Care Med 2003; 168: 1034-42.

21.- SOMFAY A, PORSZASZ J, LEE SM, CASABURI R. Dose-response effect of oxygen on hyperinflation and exercise endurance in nonhypoxaemic COPD patients. Eur Respir J 2001; 18: 77-84.

22.- FUJIMOTO K, MATSUZAWA Y, YAMAGUCHI S, KOIZUMI T, KUBO K. Benefits of oxygen on exercise performance and pulmonary hemodynamics in patients with COPD with mild hypoxemia. Chest 2002; 122: 457-63.

23.- SANTOS S, PEINADO V I, RAMÍREZ J, MELGOSA T, ROCA J, RODRÍGUEZ-ROISÍN R, et al. Characterization of pulmonary vascular remodeling in smokers and patients with mild COPD. Eur Respir J 2002; 19: 632-8.

24.- VOELKEL N F, TUDER R M. Hypoxia-induced pulmonary vascular remodeling: a model for what human disease? J Clin Invest 2000; 106: 733-8.

25.- CHETTY K G, BROWN S E, LIGHT R W. Improved exercise tolerance of the polycythemic lung patient following phlebotomy. Am J Med 1983; 74: 415-20.

26.- SIETSEMA K. Cardiovascular limitations in chronic pulmonary disease. Med Sci Sports Exerc 2001; 33: S656-61.

27.- MACNEE W. Pathophysiology of cor pulmonale in chronic obstructive pulmonary disease: part one. Am J Respir Crit Care Med 1994; 150: 833-52.

28.- BUTLER J, SCHRIJEN F, HENRÍQUEZ A, POLU J $\mathrm{M}$, ALBERT R K. Cause of the raised wedge pressure on exercise in chronic obstructive pulmonary disease. Am Rev Respir Dis 1988; 138: 350-4.

29.- CHABOT F, SCHRIJEN F, POINCELOT F, POLU J $\mathrm{M}$. Interpretation of high wedge pressure on exercise in patients with chronic obstructive pulmonary disease. Cardiology 2001; 95: 139-45.

30.- MALTAIS F, LEBLANC P, JOBIN J. Intensity of training and physiological adaptation in patients with chronic obstructive pulmonary disease. Am J Respir Crit Care Med 1997; 155: 555-61.

31.- CASABURI R, PORSZASZ J, BURNS M R, CARI- 
THERS E R, CHANG R S, COOPER C B. Physiologic benefits of exercise training in rehabilitation of patients with severe chronic obstructive pulmonary disease. Am J Respir Crit Care Med 1997; 155: 154151.

32.- PUENTE-MAESTU L, SANZ M L, SANZ P, RUIZ DE ONA J M, RODRÍGUEZ-HERMOSA J L, WHIPP

B J. Effects of two types of training on pulmonary and cardiac responses to moderate exercise in patients with COPD. Eur Respir J 2000; 15: 1026-32.

33.- SCHOLS A M, SOETERS P B, DINGEMANS A M, MOSTERT R, FRANTZEN P J, WOUTERS E F. Prevalence and characteristics of nutritional depletion in patients with stable COPD eligible for pulmonary rehabilitation. Am Rev Respir Dis 1993; 147: 1151-6.

34.- American Thoracic Society/European Respiratory Society. Skeletal muscle dysfunction in chronic obstructive pulmonary disease: a statement of the American Thoracic Society and European Respiratory Society. Am J Respir Crit Care Med 1999; 159: S1-S40.

35.- BERNARD S, LEBLANC P, WHITTOM F, CARRIER G, JOBIN J, BELLEAU R, et al. Peripheral muscle weakness in patients with chronic obstructive pulmonary disease. Am J Respir Crit Care Med 1998; 158: 629-34.

36.- FRANSSEN F M, WOUTERS E F, BAARENDS E M, AKKERMANS M A, SCHOLS A M. Arm mechanical efficiency and arm exercise capacity are relatively preserved in chronic obstructive pulmonary disease. Med Sci Sports Exerc 2002; 34: 1570-6.

37.- MALTAIS F, SIMARD A A, SIMARD C, JOBIN J, DESGAGNES P, LEBLANC P. Oxidative capacity of the skeletal muscle and lactic acid kinetics during exercise in normal subjects and in patients with COPD. Am J Respir Crit Care Med 1996; 153: 288-93.

38.- MALTAIS F, JOBIN J, SULLIVAN M J, BERNARD S, WHITTOM F, KILLIAN K J, et al. Metabolic and hemodynamic responses of lower limb during exercise in patients with COPD. J Appl Physiol 1998; 84: 1573 80.

39.- KILLIAN K J, LEBLANC P, MARTIN D H, SUMMERS E, JONES N L, CAMPBELL E J. Exercise capacity and ventilatory, circulatory, and symptom limitation in patients with chronic airflow limitation. Am Rev Respir Dis 1992; 146: 935-40.

40.- SAEY D, DEBIGARE R, LEBLANC P, MADOR M J, COTE C G, JOBIN J, et al. Contractile leg fatigue after cycle exercise: a factor limiting exercise in patients with COPD. Am J Respir Crit Care Med 2003; 168: 425-30.

41.- LEVINE S, KAISER L, LEFEROVICH J, TIKUNOV B. Cellular adaptations in the diaphragm in chronic obstructive pulmonary disease. N Engl J Med 1997; 337: 1799-806.

42.- LEVINE S, GREGORY C, NGUYEN T, SHRAGER J, KAISER L, RUBINSTEIN N, et al. Bioenergetic adap- tation of individual human diaphragmatic myofibers to severe COPD. J Appl Physiol 2002; 92: 1205-13.

43.- SIMILOWSKI T, YAN S, GAUTHIER A P, MACKLEM P T, BELLEMARE F. Contractile properties of the human diaphragm during chronic hyperinflation. $\mathrm{N}$ Engl J Med 1991; 325: 917-23.

44.- OROZCO-LEVI M, GEA J, LLORETA J L, FELEZ M, MINGUELLA J, SERRANO S, et al. Subcellular adaptation of the human diaphragm in chronic obstructive pulmonary disease. Eur Respir J 1999; 13: 371-8.

45.- DOUCET M, DEBIGARE R, JOANISSE D R, COTE C, LEBLANC P, GREGOIRE J, et al. Adaptation of the diaphragm and the vastus lateralis in mild-tomoderate COPD. Eur Respir J 2004; 24: 971-9.

46.- ROCHESTER D F, BRAUN N M. Determinants of maximal inspiratory pressure in chronic obstructive pulmonary disease. Am Rev Respir Dis 1985; 132: 42-7.

47.- PÉREZ T, BECQUART L A, STACH B, WALLAERT B, TONNEL A B. Inspiratory muscle strength and endurance in steroid-dependent asthma. Am J Respir Crit Care Med 1996; 153: 610-5.

48.- POLKEY M I, KYROUSSIS D, HAMNEGARD C H, MILLS G H, GREEN M, MOXHAM J. Diaphragm strength in chronic obstructive pulmonary disease. Am J Respir Crit Care Med 1996; 154: 1310-7.

49.- BEGIN P, GRASSINO A. Inspiratory muscle dysfunction and chronic hypercapnia in chronic obstructive pulmonary disease. Am Rev Respir Dis 1991; 143: 905-12.

50.- HAMILTON A L, KILLIAN K J, SUMMERS E, JONES N L. Muscle strength, symptom intensity, and exercise capacity in patients with cardiorespiratory disorders. Am J Respir Crit Care Med 1995; 152: 2021-31.

51.- HEIJDRA Y F, DEKHUIJZEN P N, VAN HERWAARDEN C L, FOLGERING H T. Nocturnal saturation improves by target-flow inspiratory muscle training in patients with COPD. Am J Respir Crit Care Med 1996; 153: 260-5.

52.- O'DONNELL D E, BERTLEY J C, CHAU L K, WEBB K A. Qualitative aspects of exertional breathlessness in chronic airflow limitation: pathophysiologic mechanisms. Am J Respir Crit Care Med 1997; 155: 109-15.

53.- SHEEL A W, DERCHAK P A, PEGELOW D F, DEMPSEY J A. Threshold effects of respiratory muscle work on limb vascular resistance. Am J Physiol Heart Circ Physiol 2002; 282: H1732-8.

54.- O'DONNELL D E, LAM M I U, WEBB K A. Spirometric correlates of improvement in exercise performance after anticholinergic therapy in chronic obstructive pulmonary disease. Am J Respir Crit Care Med 1999; 160: 542-9.

55.- O'DONNELL D E, FLÜGE T, GERKEN F, HAMILTON A, WEBB K, AGUILANIU B, et al. Effects 
of tiotropium on lung hyperinflation, dyspnoea and exercise tolerance in COPD. Eur Respir J 2004; 23 : 832-40.

56.- O'DONNELL D E, VODUC N, FITZPATRICK M, WEBB K A. Effect of salmeterol on the ventilatory response to exercise in chronic obstructive pulmonary disease. Eur Respir J 2004; 24: 86-94.

57.- Report of the Medical Research Council Working Party. Long term domiciliary oxygen therapy in chronic hypoxic cor pulmonale complicating chronic bronchitis and emphysema. Lancet 1981; 1: 681-6.

58.- DEAN N C, BROWN J K, HIMELMAN R B, DOHERTY J J, GOLD W M, STULBARG M S. Oxygen may improve dyspnea and endurance in patients with chronic obstructive pulmonary disease and only mild hypoxemia. Am Rev Respir Dis 1992; 146: 941-5.

59.- O'DONNELL D E, BAIN D J, WEBB K A. Factors contributing to relief of exertional breathlessness during hyperoxia in chronic airflow limitation. Am J Respir Crit Care Med 1997; 155: 530-5.

60.- PALANGE P, VALLI G, ONORATI P, ANTONUCCI R, PAOLETTI P, ROSATO A, et al. Effect of heliox on lung dynamic hyperinflation, dyspnea, and exercise endurance capacity in COPD patients. J Appl Physiol 2004; 97: 1637-42.

61.- GOTO S, PORSZASZ J, SAKURAI S, WHIPP B J, CASABURI R. Effect of helium breathing on dynamic hyperinflation, minute ventilation and exercise tolerance in severe COPD patients. Am J Respir Crit Care Med 2004; 169: A467.

62.- LACASSE Y, GUYATT G H, GOLDSTEIN R S. The components of a respiratory rehabilitation program: a systematic overview. Chest 1997; 111: 1077-88.

63.- PORSZASZ J, EMTNER M, GOTO S, SOMFAY A, WHIPP B J, CASABURI R. High intensity training decreases exercise-induced hyperinflation in patients with COPD. Chest 2005; 128: 2025-34.

64.- CASABURI R, KAKAFKA D, COOPER C, WITEK J, KESTEN S. Improvement in endurance time with the combination of tiotropium and pulmonary rehabilitation in patients with COPD. Chest 2005; 127: 809-17.

65.- SIMPSON K, KILLIAN K, MCCARTNEY N, STUBBING D G, JONES N L. Randomised controlled trial of weightlifting exercise in patients with chronic airflow limitation. Thorax 1992; 47: 70-5.

66.- DECRAMER M, GOSSELINK R, TROOSTERS T, VERSCHUEREN M, EVERS G. Muscle weakness is related to utilization of health care resources in COPD patients. Eur Respir J 1997; 10: 417-23.

67.- SWALLOW E B, REYES D, HOPKINSON N S, MAN W D, PORCHER R, CETTI E J, et al. Quadriceps strength predicts mortality in patients with moderate to severe chronic obstructive pulmonary disease. Thorax 2007; 62: 115-20.

68.- WUYAM B, PAYEN J F, LEVY P, BENSAIDANE H, REUTENAUER H, LE BAS J F, et al. Metabolism and aerobic capacity of skeletal muscle in chronic respiratory failure related to chronic obstructive pulmonary disease. Eur Respir J 1992; 5: 157-62.

69.- MAN W D, SOLIMAN M G, GEARING J, RADFORD S G, RAFFERTY G F, GRAY B J, et al. Symptoms and quadriceps fatigability after walking and cycling in chronic obstructive pulmonary disease. Am J Respir Crit Care Med 2003; 168: 562-7.

70.- MAN W D, HOPKINSON N S, HARRAF F, NIKOLETOU D, POLKEY M I, MOXHAM J. Abdominal muscle and quadriceps strength in chronic obstructive pulmonary disease. Thorax 2005; 60: 718-22.

71.- SERRES I, GAUTIER V, VARRAY A, PREFAUT C. Impaired skeletal muscle endurance related to physical inactivity and altered lung function in COPD patients. Chest 1998; 113: 900-5.

72.- ALLAIRE J, MALTAIS F, DOYON J F, NOEL M, LEBLANC P, CARRIER G, et al. Peripheral muscle endurance and the oxidative profile of the quadriceps in patients with COPD. Thorax 2004; 59: 673-8.

73.- MAN W D, SOLIMAN M G, NIKOLETOU D, HARRIS M L, RAFFERTY G F, MUSTFA N, et al. Non-volitional assessment of skeletal muscle strength in patients with chronic obstructive pulmonary disease. Thorax 2003; 58: 665-9.

74.- POLKEY M I, KYROUSSIS D, KEILTY S E, HAMNEGARD C H, MILLS G H, GREEN M, et al. Exhaustive treadmill exercise does not reduce twitch transdiaphragmatic pressure in patients with COPD. Am J Respir Crit Care Med 1995; 152: 959-64.

75.- POLKEY M I, KYROUSSIS D, HAMNEGARD C H, MILLS G H, HUGHES P D, GREEN M, et al. Diaphragm performance during maximal voluntary ventilation in chronic obstructive pulmonary disease. Am J Respir Crit Care Med 1997; 155: 642-8.

76.- STUBBINGS A K, MOORE A J, DUSMET M, GOLDSTRAW P, WEST T G, POLKEY M I, et al. Physiological properties of human diaphragm muscle fibres and the effect of chronic obstructive pulmonary disease. J Physiol 2008; 586: 2637-50.

77.- MARQUIS K, DEBIGARE R, LACASSE Y, LEBLANC P, JOBIN J, CARRIER G, et al. Midthigh muscle cross-sectional area is a better predictor of mortality than body mass index in patients with chronic obstructive pulmonary disease. Am J Respir Crit Care Med 2002; 166: 809-13.

78.- GOSSELINK R, TROOSTERS T, DECRAMER M. Peripheral muscle weakness contributes to exercise limitation in COPD. Am J Respir Crit Care Med 1996; 153: 976-80.

79.- JAKOBSSON P, JORFELDT L, BRUNDIN A. Skeletal muscle metabolites and fibre types in patients with advanced chronic obstructive pulmonary disease (COPD), with and without chronic respiratory failure. Eur Respir J 1990; 3: 192-6.

80.- HILDEBRAND I L, SYLVEN C, ESBJORNSSON 
M, HELLSTROM K, JANSSON E. Does chronic hypoxaemia induce transformations of fibre types? Acta Physiol Scand 1991; 141: 435-9.

81.- GOSKER H R, VAN MAMEREN H, VAN DIJK P J, ENGELEN M P, VAN DER VUSSE G J, WOUTERS E F. Skeletal muscle fibre-type shifting and metabolic profile in patients with chronic obstructive pulmonary disease. Eur Respir J 2002; 19: 617-25.

82.- JOBIN J, MALTAIS F, DOYON J F, LEBLANC P, SIMARD P M, SIMARD A A, et al. Chronic obstructive pulmonary disease: capillarity and fiber-type characteristics of skeletal muscle. J Cardiopulm Rehabil 1998; 18: 432-7.

83.- JAKOBSSON P, JORFELDT L, HENRIKSSON J. Metabolic enzyme activity in the quadriceps femoris muscle in patients with severe chronic obstructive pulmonary disease. Am J Respir Crit Care Med 1995; 151: 374-7.

84.- SWALLOW E B, GOSKER H R, WARD K A, MOORE A J, DAYER M J, HOPKINSON N S, et al. A novel technique for nonvolitional assessment of quadriceps muscle endurance in humans. J Appl Physiol 2007; 103: 739-46.

85.- POLKEY M I, HAWKINS P, KYROUSSIS D, ELLUM S G, SHERWOOD R, MOXHAM J. Inspiratory pressure support prolongs exercise induced lactataemia in severe COPD. Thorax 2000; 55: 547-9.

86.- THOMPSON C H, DAVIES R J, KEMP G J, TAYLOR D J, RADDA G K, RAJAGOPALAN B. Skeletal muscle metabolism during exercise and recovery in patients with respiratory failure. Thorax 1993; 48: 486-90.

87.- CAQUELARD F, BURNET H, TAGLIARINI F, CAUCHY E, RICHALET J P, JAMMES Y. Effects of prolonged hypobaric hypoxia on human skeletal muscle function and electromyographic events. Clin Sci 2000; 98: 329-37.

88.- HOPPELER H, KLEINERT E, SCHLEGEL C, CLAASSEN H, HOWALD H, KAYAR S R, et al. Morphological adaptations of human skeletal muscle to chronic hypoxia. Int J Sports Med 1990; 11 (Suppl 1): S3-S9.

89.- HOWALD H, PETTE D, SIMONEAU J A, UBER A, HOPPELER H, CERRETELLI P. Effect of chronic hypoxia on muscle enzyme activities. Int J Sports Med 1990; 11 (Suppl 1): S10-S14.

90.- PASTORIS O, DOSSENA M, FOPPA P, ARNABOLDI R, GORINI A, VILLA R F, et al. Modifications by chronic intermittent hypoxia and drug treatment on skeletal muscle metabolism. Neurochem Res 1995; 20: 143-50.

91.- SEMPLE P D, BEASTALL G H, WATSON W S, HUME R. Serum testosterone depression associated with hypoxia in respiratory failure. Clin Sci 1980; 58 105-6.

92.- TAKABATAKE N, NAKAMURA H, ABE S, INOUE
$\mathrm{S}$, HINO T, SAITO H, et al. The relationship between chronic hypoxemia and activation of the tumor necrosis factor- $\alpha$ system in patients with chronic obstructive pulmonary disease. Am J Respir Crit Care Med 2000; 161: 1179-84.

93.- HEUNKS L M, VINA J, VAN HERWAARDEN C L, FOLGERING H T, GIMENO A, DEKHUIJZEN P N. Xanthine oxidase is involved in exercise-induced oxidative stress in chronic obstructive pulmonary disease. Am J Physiol 1999; 277: R1697-R1704.

94.- PAYEN J F, WUYAM B, LEVY P, REUTENAUER H, STIEGLITZ P, PARAMELLE B, et al. Muscular metabolism during oxygen supplementation in patients with chronic hypoxemia. Am Rev Respir Dis 1993; 147: 592-8.

95.- GERTZ I, HEDENSTIERNA G, HELLERS G, WAHREN J. Muscle metabolism in patients with chronic obstructive lung disease and acute respiratory failure. Clin Sci Mol Med 1977; 52: 396-403.

96.- POUW E M, SCHOLS A M, VAN DER VUSSE G J, WOUTERS E F. Elevated inosine monophosphate levels in resting muscle of patients with stable chronic obstructive pulmonary disease. Am J Respir Crit Care Med 1998; 157: 453-7.

97.- JUAN G, CALVERLEY P, TALAMO C, SCHNADER J, ROUSSOS C. Effect of carbon dioxide on diaphragmatic function in human beings. N Engl J Med 1984; 310: 874-9.

98.- VIANNA L G, KOULOURIS N, LANIGAN C, MOXHAM J. Effect of acute hypercapnia on limb muscle contractility in humans. J Appl Physiol 1990; 69: 148693.

99.- WEINER P, AZGAD Y, WEINER M. The effect of corticosteroids on inspiratory muscle performance in humans. Chest 1993; 104: 1788-91.

100.- MILLS G H, KYROUSSIS D, JENKINS P, HAMNEGARD C H, POLKEY M I, WASS J, et al. Respiratory muscle strength in Cushing's syndrome. Am J Respir Crit Care Med 1999; 160: 1762-5.

101.- DECRAMER M, LACQUET L M, FAGARD R, ROGIERS P. Corticosteroids contribute to muscle weakness in chronic airflow obstruction. Am J Respir Crit Care Med 1994; 150: 11-16.

102.- ENGELEN M P, SCHOLS A M, BAKEN W C, WESSELING G J, WOUTERS E F. Nutritional depletion in relation to respiratory and peripheral skeletal muscle function in out-patients with COPD. Eur Respir J 1994; 7 : 1793-7.

103.- LOPES J, RUSSELL D M, WHITWELL J, JEEJEEBHOY K N. Skeletal muscle function in malnutrition. Am J Clin Nutr 1982; 36: 602-10.

104.- FERREIRA I M, BROOKS D, LACASSE Y, GOLDSTEIN R S. Nutritional supplementation in stable chronic obstructive pulmonary disease. Cochrane Database Syst. Rev. CD000998, doi:10.1002/14651858. CD000998.pub2. 
105.- DEBIGARE R, MARQUIS K, COTE C H, TREMBLAY R R, MICHAUD A, LEBLANC P, et al. Catabolic/anabolic balance and muscle wasting in patients with COPD. Chest 2003; 124: 83-9.

106.- KAMISCHKE A, KEMPER D E, CASTEL M A, LUTHKE M, ROLF C, BEHRE H M, et al. Testosterone levels in men with chronic obstructive pulmonary disease with or without glucocorticoid therapy. Eur Respir J 1998; 11: 41-5.

107.- BURDET L, DE MURALT B, SCHUTZ Y, PICHARD C, FITTING J W. Administration of growth hormone to underweight patients with chronic obstructive pulmonary disease. A prospective, randomized, controlled study. Am J Respir Crit Care Med 1997; 156: 1800-6.

108.- SCHOLS A M, SOETERS P B, MOSTERT R, PLUYMERS R J, WOUTERS E F. Physiologic effects of nutritional support and anabolic steroids in patients with chronic obstructive pulmonary disease. A placebo-controlled randomized trial. Am J Respir Crit Care Med 1995; 152: 1268-74.

109.- CREUTZBERG E C, SCHOLS A M, WELINGSCHEEPERS C A, BUURMAN W A, WOUTERS E F. Characterization of nonresponse to high caloric oral nutritional therapy in depleted patients with chronic obstructive pulmonary disease. Am J Respir Crit Care Med 2000; 161: 745-52.

110.- DI FRANCIA M, BARBIER D, MEGE J L, OREHEK J. Tumor necrosis factor- $\alpha$ levels and weight loss in chronic obstructive pulmonary disease. Am J Respir Crit Care Med 1994; 150: 1453-5.

111.- SCHOLS A M, BUURMAN W A, STAAL VAN DEN BREKEL A J, DENTENER M A, WOUTERS E F. Evidence for a relation between metabolic derangements and increased levels of inflammatory mediators in a subgroup of patients with chronic obstructive pulmonary disease. Thorax 1996; 51: 819-24.

112.- SPRUIT M A, GOSSELINK R, TROOSTERS T, KASRAN A, GAYAN-RAMÍREZ G, BOGAERTS $\mathrm{P}$, et al. Muscle force during an acute exacerbation in hospitalized patients with COPD and its relationship with CXCL8 and IGF-1. Thorax 2003; 58: 752-6.

113.- BARREIRO E, SCHOLS A M, POLKEY M I, GALDIZ J B, GOSKER H R, SWALLOW E B, et al. Cytokine profile in quadriceps muscles of patients with severe COPD. Thorax 2008; 63: 100-7.

114.- RAHMAN I, SKWARSKA E, MAC NEE W. Attenuation of oxidant/antioxidant imbalance during treatment of exacerbations of chronic obstructive pulmonary disease. Thorax 1997; 52: 565-8.

115.- COUILLARD A, MALTAIS F, SAEY D, DEBIGARE R, MICHAUD A, KOECHLIN C, et al. Exerciseinduced quadriceps oxidative stress and peripheral muscle dysfunction in patients with chronic obstructive pulmonary disease. Am J Respir Crit Care Med 2003; 167: 1664-9.

116.- KOECHLIN C, COUILLARD A, SIMAR D, CRIS-
TOL J P, BELLET H, HAYOT M, et al. Does oxidative stress alter quadriceps endurance in chronic obstructive pulmonary disease? Am J Respir Crit Care Med 2004; 169: 1022-7.

117.- ARDEN N K, SPECTOR T D. Genetic influences on muscle strength, lean body mass, and bone mineral density: a twin study. J Bone Miner Res 1997; 12: 2076-81.

118.- HOPKINSON N S, NICKOL A H, PAYNE J, HAWE E, MAN W D, MOXHAM J, et al. Angiotensin converting enzyme genotype and strength in chronic obstructive pulmonary disease. Am J Respir Crit Care Med 2004; 170: 395-9.

119.- HOPKINSON N S, LI K W, KEHOE A, HUMPHRIES S E, ROUGHTON M, MOXHAM J, et al. Vitamin D receptor genotypes influence quadriceps strength in chronic obstructive pulmonary disease. Am J Clin Nutr 2008; 87: 385-90.

120.- PITTA F, TROOSTERS T, SPRUIT M A, PROBST V S, DECRAMER M, GOSSELINK R. Characteristics of physical activities in daily life in chronic obstructive pulmonary disease. Am J Respir Crit Care Med 2005; 171: 972-7.

121.- SINGH S, MORGAN M D. Activity monitors can detect brisk walking in patients with chronic obstructive pulmonary disease. J Cardiopulm Rehabil 2001; 21: 143-8.

122.- WALKER P P, BURNETT A, FLAVAHAN P W, CALVERLEY P M. Lower limb activity and its determinants in COPD. Thorax 2008; 63: 683-9.

123.- GARCÍA-AYMERICH J, LANGE P, BENET M, SCHNOHR P, ANTO J M. Regular physical activity modifies smoking-related lung function decline and reduces risk of chronic obstructive pulmonary disease: a population-based cohort study. Am J Respir Crit Care Med 2007; 175: 458-63.

124.- GARCÍA-AYMERICH J, LANGE P, BENET M, SCHNOHR P, ANTO J M. Regular physical activity reduces hospital admission and mortality in chronic obstructive pulmonary disease: a population based cohort study. Thorax 2006; 61: 772-8.

125.- FRANSSEN F M, WOUTERS E F, SCHOLS A M. The contribution of starvation, deconditioning and ageing to the observed alterations in peripheral skeletal muscle in chronic organ diseases. Clin Nutr 2002; 21 : $1-14$. 